\title{
Laser-assisted Mott scattering in the Coulomb approximation
}

\author{
S-M Li ${ }^{1,2}, \mathbf{J ~ B e r a k d a r}^{2}, \mathrm{~J}^{\text {Chen }}{ }^{3}$ and Z-F Zhou ${ }^{1}$ \\ ${ }^{1}$ Open Laboratory of Bond Selective Chemistry, Department of Modern Physics, \\ University of Science and Technology of China, PO Box 4, Hefei, Anhui 230027, \\ People's Republic of China \\ ${ }^{2}$ Max-Planck Institut für Mikrostrukturphysik, Weinberg 2, 06120 Halle, Germany \\ ${ }^{3}$ China Center of Advanced Science and Technology (World Laboratory), PO Box 8730, \\ Beijing 100080, People's Republic of China \\ E-mail: jber@mpi-halle.de
}

Received 28 August 2003, in final form 17 November 2003

Published 23 January 2004

Online at stacks.iop.org/JPhysB/37/653 (DOI: 10.1088/0953-4075/37/3/010)

\begin{abstract}
We investigate the influence of the Coulomb interaction on laser-assisted Mott scattering. This is done by using an approximation in which the initial state of the electron is described by a Coulomb function modulated by the laser in the same way as a relativistic Volkov state. Numerical calculations are carried out for medium field intensity, and compared with the corresponding results obtained within the first-order Born approximation (where Coulomb distortion effects are neglected). It is found that the differential cross sections of the present treatment agree well with the Born predictions at small angles. With increasing scattering angles, however, including the Coulomb interaction enhances the cross section, as compared to the first-Born results. Furthermore, we show that the Coulomb distortion of the electron motion leads to a qualitatively different dependence of the cross sections on the laser frequency at high frequencies. The dependences of the differential cross section on the other field parameters, impact energy and the charge state of the residual ion are also studied.
\end{abstract}

(Some figures in this article are in colour only in the electronic version)

\section{Introduction}

The availability of ultra-intense lasers [1] and high-energy electron [2] beams opened the way for the investigation of laser-assisted electron-nucleus scattering in the relativistic regime. These advances have triggered a renewed interest in the theoretical aspects of this process. Here we mention several theoretical studies on laser-assisted Mott scattering which are of direct relevance to the present work: based on the theory of Denisov and Fedorov [3] the relativistic 
potential scattering in the presence of a strong laser field has been investigated in the first Born approximation (FBA) [4-7]. Rosenberg and co-worker [8] studied the laser-assisted relativistic scattering of a charged particle by a force centre using variational approaches. Roshchupkin [9] developed a second-order Born approximation to analyse the relativistic scattering from a Coulomb potential. Hovhannisyan et al [10] presented a treatment of the scattering by an atomic target at small angles in the eikonal approximation. This was done by modelling the atomic scattering potential by a screened Coulomb potential with a nuclear charge $Z=1$ au and a screening length $\ell=4$ au.

All the above treatments are expected to perform satisfactorily in low order approximations or at small scattering angles. For large scattering angles, however, the consideration of higher order corrections is necessary, but this is a difficult problem with no known analytical solution. In contrast, the cross section for laser-free, non-relativistic scattering from a pure Coulomb potential is known in closed analytical form. In this paper, we propose a method to treat the laser-assisted scattering of a relativistic electron from the Coulomb field of a bare nucleus (Mott scattering), and refer to it as the Coulomb approximation (CA). Within the approximation specified below, an analytical expression for the laser-modified cross section is obtained. The physical content of this approximation and the consequences of including the Coulomb interaction in the theory are demonstrated by numerical calculations of the cross section at various field parameters and for different electron energies and charge states of target.

This paper is organized as follows: in section 2, we derive the CA scattering matrix elements and cross sections. In section 3, we present numerical results and compare them with the results obtained by the FBA. The main conclusions and the validity of the approximation are briefly summarized in section 4 . The Minkowski metric tensor $g_{\mu \nu}=\operatorname{diag}(1,-1,-1,-1)$ and atomic units $\hbar=m=e=1$ are used throughout.

\section{Theory}

For simplicity, we treat the laser field as a linearly polarized classical electromagnetic field described by the four potential

$$
a(\varphi)=A \cos \varphi,
$$

where $A=(0, \mathbf{A}), \mathbf{A}$ is the amplitude of the vector potential, and $\varphi=k \cdot x, k=\left(\frac{\omega}{c}, \mathbf{k}\right)$ is the four-wave number. The $S$-matrix (prior form) for the electron-nucleus scattering assisted by such a laser field is

$$
S_{f i}=\mathrm{i} \frac{Z}{c} \int \mathrm{d}^{4} x \bar{\psi}_{f} \frac{\gamma_{0}}{|\mathbf{x}|} \psi_{i}^{(+)}
$$

Here $Z$ is the charge of the target, $\bar{\psi}_{f}=\psi_{f}^{\dagger} \gamma_{0}$, and $\psi_{f}$ is the outgoing state of the electron which is described by the relativistic Volkov wavefunction normalized in the volume $V$ [11], i.e.

$$
\begin{aligned}
\psi_{f} & =\left[1-\frac{k \phi t}{2 c\left(k \cdot p^{\prime}\right)}\right] \frac{u^{\prime}}{\sqrt{2 Q^{\prime} V}} \exp \left[-\mathrm{i} q^{\prime} \cdot x+\mathrm{i} \int^{k \cdot x} \frac{p^{\prime} \cdot a(\varphi)}{c\left(k \cdot p^{\prime}\right)} \mathrm{d} \varphi\right], \\
& =\left[1-\frac{k \phi}{2 c\left(k \cdot p^{\prime}\right)}\right] \frac{u^{\prime}}{\sqrt{2 Q^{\prime} V}} \exp \left[-\mathrm{i} q^{\prime} \cdot x+\mathrm{i} \frac{p^{\prime} \cdot A}{c\left(k \cdot p^{\prime}\right)} \sin (k \cdot x)\right] .
\end{aligned}
$$

The prime is used as a super index to label the quantities of the final state electron. $u^{\prime}$ represents a bispinor for the free electron which satisfies the Dirac equation in the absence of the laser, and which is normalized as $\bar{u}^{\prime} u^{\prime}=u^{\prime \dagger} \gamma_{0} u^{\prime}=2 c^{2}$. The notation $\psi$ for a certain four-vector $v$ 
means the multiplication by the Dirac matrix $\gamma^{\mu}$ while summing over the index. $q^{\prime}=\left(\frac{Q^{\prime}}{c}, \mathbf{q}^{\prime}\right)$ is the averaged four-momentum of the electron inside the laser field with

$$
q^{\prime \mu}=p^{\prime \mu}-\frac{\bar{a}^{2}}{2 c^{2}\left(k \cdot p^{\prime}\right)} k^{\mu},
$$

where $\bar{a}^{2}$ is the time average of $a^{2}$. In equation (2) $\psi_{i}^{(+)}$describes the initial state of the electron when subjected to the simultaneously influence of the laser and the Coulomb field of the target nucleus. No exact (analytical) solution is available for $\psi_{i}^{(+)}$. In this work we approximate this state by a Coulomb wavefunction [11] phase-modulated by the laser in the same way as the relativistic Volkov state. Specifically we use

$$
\begin{aligned}
& \psi_{i}^{(+)}=G_{1} F_{1}(\mathrm{i} \xi,1, \mathrm{i}(|\mathbf{q} \| \mathbf{x}|-\mathbf{q} \cdot \mathbf{x}))\left[1-\frac{\not k \phi}{2 c(k \cdot p)}\right] \frac{u}{\sqrt{2 Q V}} \\
& \times \exp \left[-\mathrm{i} q \cdot x+\mathrm{i} \frac{p \cdot A}{c(k \cdot p)} \sin (k \cdot x)\right],
\end{aligned}
$$

where $\xi=Z \alpha \frac{Q}{c|\mathbf{q}|}$ is the relativistic Sommerfeld parameter and $\alpha$ is the fine structure constant. $G=\mathrm{e}^{\pi \xi / 2} \Gamma(1-\mathrm{i} \xi)$ is a normalization factor and ${ }_{1} F_{1}(a, b, c)$ is the confluent hypergeometric function in the notation of [12].

To obtain analytical results we proceed as follows. We insert equations (3) and (5) into the formula for the $S$-matrix (given by equation (2)) and exploit the expansion

$$
\mathrm{e}^{\mathrm{i} y \sin u}=\sum_{l=-\infty}^{\infty} J_{l}(y) \mathrm{e}^{\mathrm{i} l u}
$$

With the aid of the formula for the Bessel functions [13]

$$
J_{l-1}(y)+J_{l+1}(y)=\frac{2 l}{y} J_{l}(y)
$$

we can then perform the integration over the time variable in the scattering matrix and obtain

$$
S_{f i}=\mathrm{i} Z G \sum_{l=-\infty}^{+\infty}\left\{\frac{I}{\sqrt{4 Q Q^{\prime} V^{2}}} \bar{u}^{\prime} f u 2 \pi \delta\left(Q^{\prime}-Q+l \omega\right)\right\}
$$

where $[14,15]$

$I=\int \mathrm{d}^{3} x \frac{\mathrm{e}^{\mathrm{i}\left(\mathbf{q}^{\prime}-\mathbf{q}+l \mathbf{k}\right) \cdot \mathbf{x}}}{|\mathbf{x}|}{ }_{1} F_{1}(\mathrm{i} \xi, 1, \mathrm{i}(|\mathbf{q} \| \mathbf{x}|-\mathbf{q} \cdot \mathbf{x}))=4 \pi \frac{\left[\left(\mathbf{q}^{\prime}+l \mathbf{k}\right)^{2}-\mathbf{q}^{2}\right]^{-\mathrm{i} \xi}}{\left[\left(\mathbf{q}^{\prime}-\mathbf{q}+l \mathbf{k}\right)^{2}\right]^{1-\mathrm{i} \xi}}$

and

$$
f=\Delta_{0} \gamma_{0}+\Delta_{1} \gamma_{0} k A+\Delta_{2} A k \gamma_{0}+\Delta_{3} A k \gamma_{0} k A .
$$

Here we have introduced the abbreviations

$$
\begin{aligned}
& \Delta_{0}=J_{l}(D), \\
& \Delta_{1}=-\frac{l J_{l}(D)}{2 c(k \cdot p) D}, \\
& \Delta_{2}=-\frac{l J_{l}(D)}{2 c\left(k \cdot p^{\prime}\right) D}, \\
& \Delta_{3}=\frac{J_{l-2}(D)+2 J_{l}(D)+J_{l+2}(D)}{16 c^{2}(k \cdot p)\left(k \cdot p^{\prime}\right) D},
\end{aligned}
$$

where $J_{l}(D)$ is the ordinary Bessel function with the argument $D=\frac{p \cdot A}{c(k \cdot p)}-\frac{p^{\prime} \cdot A}{c\left(k \cdot p^{\prime}\right)}$. 
The differential cross section $\mathrm{d} \sigma$ for the laser-assisted free-free transition is proportional to the modulus square of $S_{f i}$, to the density of final states $\left|q^{\prime}\right\rangle$ in phase space divided by the time $T$ and to the incoming flux of electrons [4]. Hence we write

$\mathrm{d} \sigma=\frac{\left|S_{f i}\right|^{2}}{T}\left(\frac{V \mathrm{~d}^{3} q^{\prime}}{8 \pi^{3}}\right) \frac{Q V}{c^{2}|\mathbf{q}|}=\frac{Z^{2}|G|^{2}}{16 \pi^{2} c^{4}} \sum_{l=-\infty}^{\infty}\left\{|I|^{2}\left|\bar{u}^{\prime} f u\right|^{2} \delta\left(Q^{\prime}-Q+l \omega\right) \frac{\left|\mathbf{q}^{\prime}\right|}{|\mathbf{q}|} \mathrm{d} Q^{\prime} \mathrm{d} \Omega\right\}$.

Integrating over the final state energy $Q^{\prime}$, we obtain the cross section differential in the solid angle $\Omega$ of the scattered electron

$$
\frac{\mathrm{d} \sigma}{\mathrm{d} \Omega}=\frac{Z^{2}|G|^{2}}{16 \pi^{2} c^{4}} \sum_{l=-\infty}^{\infty} \frac{\left|\mathbf{q}^{\prime}\right|}{|\mathbf{q}|}|I|^{2}\left|\bar{u}^{\prime} f u\right|^{2} .
$$

Usually the experiments cannot resolve either the initial or the final spin polarizations. Thus, it is of interest to inspect the spin-unpolarized cross section $\frac{\mathrm{d} \bar{\sigma}}{\mathrm{d} \Omega}$ which is obtained by summing equation (16) over the final spin states and averaging over the initial spin polarizations [16], i.e.

$$
\frac{\mathrm{d} \bar{\sigma}}{\mathrm{d} \Omega}=\frac{Z^{2}|G|^{2}}{16 \pi^{2} c^{4}} \sum_{l=-\infty}^{\infty} \frac{\left|\mathbf{q}^{\prime}\right|}{|\mathbf{q}|}|I|^{2} \frac{1}{2} \sum_{s s^{\prime}}\left|\bar{u}^{\prime} f u\right|^{2} .
$$

The sum over the spins can be evaluated with help of the well-known identity

$$
\sum_{s s^{\prime}}\left|\bar{u}^{\prime} f u\right|^{2}=\operatorname{Tr}\left[f\left(\not p c+c^{2}\right) \gamma^{0} f^{\dagger} \gamma^{0}\left(\not p^{\prime} c+c^{2}\right)\right] .
$$

By utilizing this relation we obtain the final expression

$$
\frac{\mathrm{d} \bar{\sigma}}{\mathrm{d} \Omega}=\sum_{l=-\infty}^{\infty} \frac{\mathrm{d} \bar{\sigma}_{l}}{\mathrm{~d} \Omega}
$$

where

$$
\frac{\mathrm{d} \bar{\sigma}_{l}}{\mathrm{~d} \Omega}=\frac{Z^{2}|G|^{2}}{8 \pi^{2} c^{2}} \frac{\left|\mathbf{q}^{\prime}\right|}{|\mathbf{q}|}|I|^{2} M
$$

The function $M$ has the same form as in [7]

$$
\begin{aligned}
M=\frac{1}{4} \operatorname{Tr}[f(\not p & \left.+c) \gamma_{0} f^{\dagger} \gamma_{0}\left(\not p^{\prime}+c\right)\right]=c^{2}\left[\Delta_{0}^{2}+4\left(\Delta_{1} \Delta_{2}-\Delta_{0} \Delta_{3}\right) A^{2} k_{0}^{2}\right] \\
& +\Delta_{0}^{2}\left(2 p_{0} p_{0}^{\prime}-p \cdot p^{\prime}\right)+\Delta_{1}^{2}\left\{2 A^{2}\left[(k \cdot p)\left(k \cdot p^{\prime}\right)-2 k_{0} p_{0}^{\prime}(k \cdot p)\right]\right\} \\
& +\Delta_{2}^{2}\left\{2 A^{2}\left[(k \cdot p)\left(k \cdot p^{\prime}\right)-2 k_{0} p_{0}\left(k \cdot p^{\prime}\right)\right]\right\}+\Delta_{3}^{2}\left[8 A^{4} k_{0}^{2}(k \cdot p)\left(k \cdot p^{\prime}\right)\right] \\
& +2 \Delta_{0} \Delta_{1}\left[2 k_{0} p_{0}^{\prime}(A \cdot p)+(k \cdot p)\left(A \cdot p^{\prime}\right)-\left(k \cdot p^{\prime}\right)(A \cdot p)\right]+2 \Delta_{0} \Delta_{2}\left[2 k_{0} p_{0}\left(A \cdot p^{\prime}\right)\right. \\
& \left.+\left(k \cdot p^{\prime}\right)(A \cdot p)-(k \cdot p)\left(A \cdot p^{\prime}\right)\right]+2 \Delta_{0} \Delta_{3}\left\{2 A ^ { 2 } k _ { 0 } \left[k_{0}\left(p \cdot p^{\prime}\right)-p_{0}\left(k \cdot p^{\prime}\right)\right.\right. \\
& \left.\left.-p_{0}^{\prime}(k \cdot p)\right]\right\}+2 \Delta_{1} \Delta_{2}\left\{4 k_{0}^{2}(A \cdot p)\left(A \cdot p^{\prime}\right)+2 A^{2}\left[k_{0} p_{0}\left(k \cdot p^{\prime}\right)\right.\right. \\
& \left.\left.+k_{0} p_{0}^{\prime}(k \cdot p)-k_{0}^{2}\left(p \cdot p^{\prime}\right)-(k \cdot p)\left(k \cdot p^{\prime}\right)\right]\right\}+2 \Delta_{1} \Delta_{3}\left[-4 A^{2} k_{0}^{2}(k \cdot p)\left(A \cdot p^{\prime}\right)\right] \\
& +2 \Delta_{2} \Delta_{3}\left[-4 A^{2} k_{0}^{2}\left(k \cdot p^{\prime}\right)(A \cdot p)\right] .
\end{aligned}
$$

In equation (20), the Coulomb modification is reflected in the factor $G$ and the integral $I$ of equation (9). If we neglect the distortion of the Coulomb field, i.e. if we set the Sommerfeld parameter $\xi=0$, we recover the corresponding expression obtained within the FBA. 


\section{Results and discussion}

In this section we analyse the numerical results for the Mott scattering assisted by a linearly polarized laser field in the $\mathrm{CA}$, and make a comparison with the corresponding results obtained within the FBA [7]. As in [7], we choose the origin of the frame of reference to coincide with the target which is supposed to be an infinitely massive nucleus. The $z$-axis lies in the direction of the incident momentum $\mathbf{p}$ of the impact electron, the $y$-axis is chosen along the direction of the field wave vector $\mathbf{k}$, and the electric field vector $\mathcal{E}_{0}$ is supposed to be in the $x z$-plane.

In figure 1 we display the multiphoton cross sections (given by equation (20)) at a scattering angle of $90^{\circ}$ versus the net number of photons transferred between the colliding system and the background laser field. The scattering geometry is such that the laser polarization is parallel to the incident momentum. It is found that accounting for the Coulomb distortion effects on the initial state leads to an enhancement of the cross sections, as compared to the cross sections obtained within the FBA. The cross section enhancements for photoemission $(l>0)$ are stronger than those for photoabsorption $(l<0)$, but the latter cover a much larger photon number range $l$.

In figure 2 the summed differential cross sections, as obtained from equation (19), are depicted. At small scattering angles, all four curves merge together, which suggests that the Coulomb field modifications are negligible and the FBA delivers satisfactory result. We recall that the laser field considered here is of a medium strength $\left(\mathcal{E}_{0}=0.01 \mathrm{au}=5.14 \times 10^{7} \mathrm{~V} \mathrm{~cm}^{-1}\right)$ and that small angle scattering events correspond to collisions at large impact parameters for which case the influence of the nucleus field on the initial electron state is less prominent. With increasing scattering angle, however, the incorporation of the Coulomb field effects of the residual ion results in cross sections that are larger than the FBA cross sections. This behaviour is valid for the laser-free and for the laser-modified case. From a physical point of view this behaviour is comprehensible since scattering through large angles requires a close encounter with the charged ion. In this case the Coulomb distortion of the initial state $\psi_{i}^{(+)}$ becomes significant.

Figure 3 shows the summed cross section versus the azimuthal angle of the scattered electron. Although the present results are higher than those yielded by the FBA, the curve varying tendencies in both approximations are more or less the same for almost all angles except for $\phi=270^{\circ}$, at which the large discrepancies between both approximations are supposed to be caused by the singularity feature of the integral in equation (9), and hence we judge that it is not physical. At $\phi=90^{\circ}$ the laser-modified cross sections in both approximations coincide with the corresponding results in the laser-free case.

Figures 4-6 are respectively the summed cross section dependencies on the field strength $\mathcal{E}_{0}$, the polarization direction $\Theta$ and the impact energy $T_{i}$ at the scattering angle $\theta=90^{\circ}$. In these figures, the $\mathrm{CA}$ results both in the laser-free and in the laser-modified cases are larger than the corresponding results obtained by means of the FBA. Although the shapes of the FBA and CA cross sections are quite similar, the effect of the Coulomb field of the residual ion cannot be modelled by simply scaling up the FBA results by a constant factor. The enhancement of the cross section when the Coulomb distortion is incorporated is understandable due to enlargement (by the Coulomb field) in the scattering region where energy and momentum can be exchanged.

In figure 7 the summed cross section is plotted against the field frequency. The general tendencies are the same in both the Coulomb and the first Born approximations. However, for the CA calculation, the laser-modified curve merges with the results for the laser-free case at much higher frequencies of the laser field than the FBA results do. The reason for this 

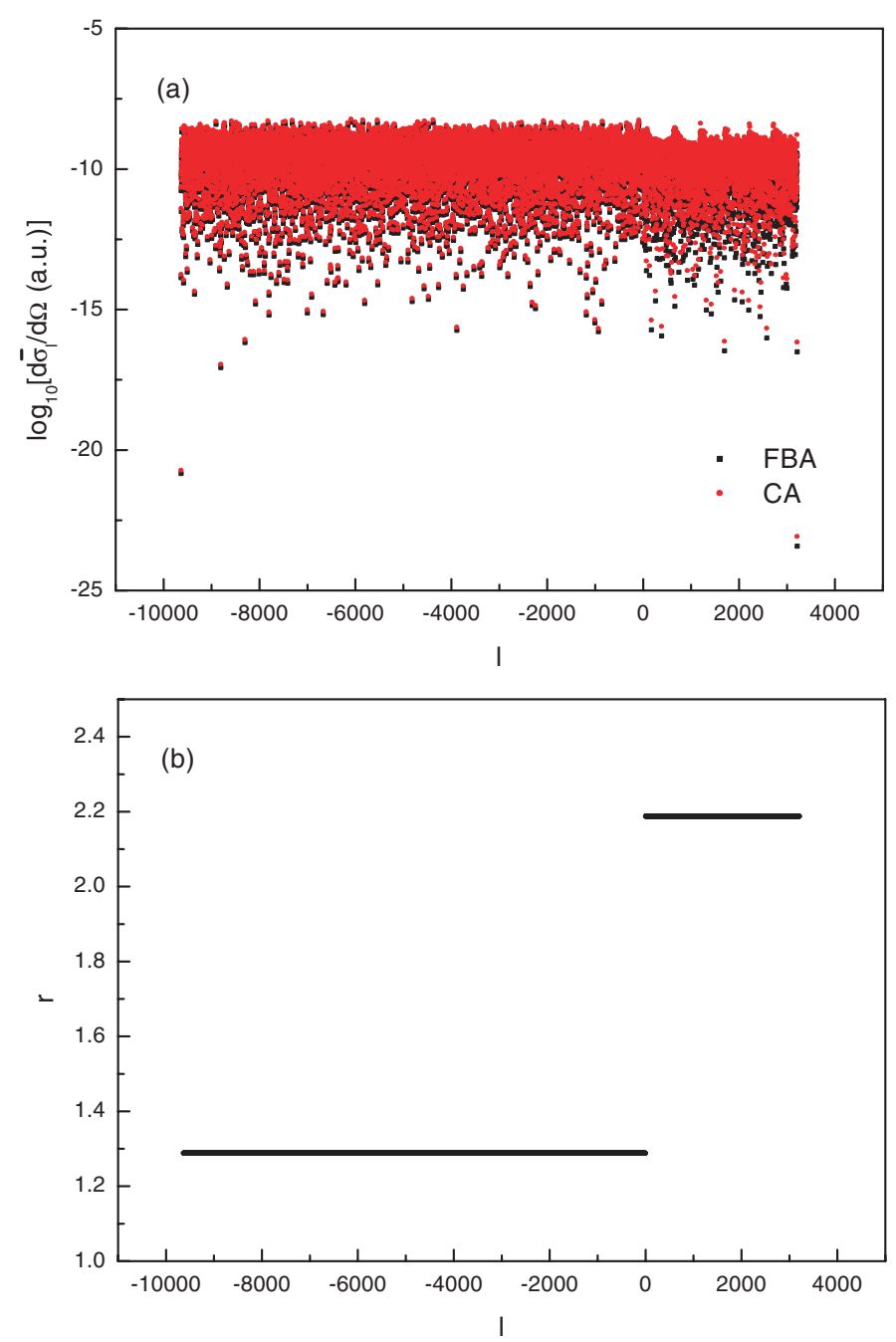

Figure 1. The multiphoton cross section versus the number of photons $l$ that are exchanged between the colliding system and the laser field. The scattering angle is $90^{\circ}$ and the electron-impact energy is $T_{i}=m c^{2}=0.511 \mathrm{MeV}$. (a) The values of multiphoton cross sections in the Coulomb (CA) and the first-Born approximations (FBA); (b) the ratio of the results of CA to those of FBA. The laser is linearly polarized along the incident direction of the electron $\mathcal{E}_{0} \| \mathbf{p}$, with a field strength $\mathcal{E}_{0}=0.01 \mathrm{au}=5.14 \times 10^{7} \mathrm{~V} \mathrm{~cm}^{-1}$. The photon energy (the field frequency) is $\hbar \omega=1.17 \mathrm{eV}$.

behaviour is that the laser-field properties determine the behaviour of the Coulomb-Volkov wavefunction of equation (5), e.g. through the frequency-dependence of the four-momentum $q$ (see equation (4)) which enters the normalization factor $G$ and the argument of the confluent hypergeometric function ${ }_{1} F_{1}(\mathrm{i} \xi, 1, \mathrm{i}(|\mathbf{q} \| \mathbf{x}|-\mathbf{q} \cdot \mathbf{x}))$.

Figure 8 illustrates the dependence of the cross sections on the charge state $Z$ of the target nucleus. As is to be expected, increasing the nucleus charge $Z$ enhances the strength of the Coulomb field of the ion. Consequently, the deviations between the CA and the FBA results become more serious for large $Z$. The magnitude of the difference between the FBA and the $\mathrm{CA}$ cross sections has to be deduced from the full numerical calculations since in the CA the 

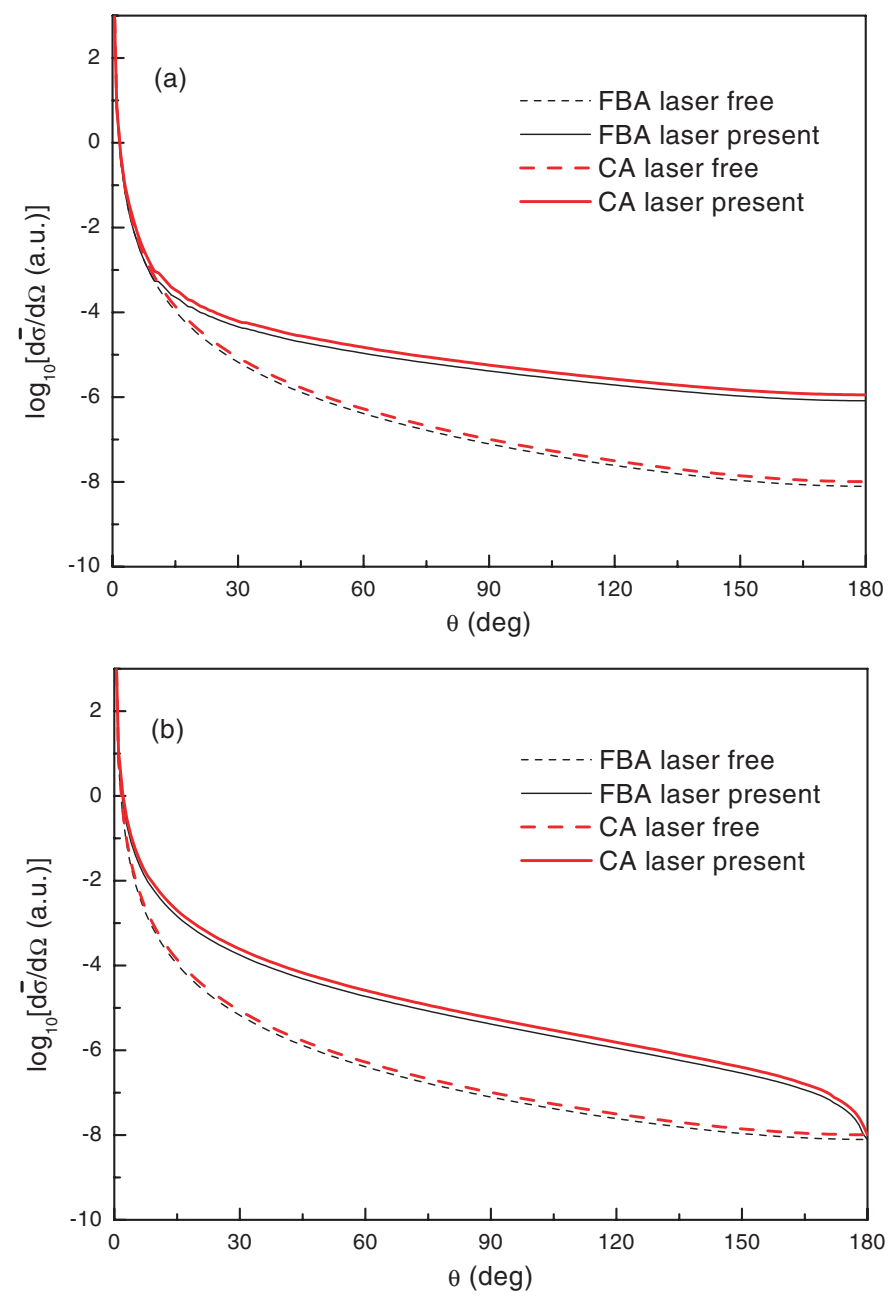

Figure 2. The differential cross sections summed over all multiphoton processes for the following laser polarization geometries: (a) $\mathcal{E}_{0} \| \mathbf{p}$ and (b) $\mathcal{E}_{0} \perp \mathbf{p}$. The azimuth angle is $\phi=0^{\circ}$. The impact energy and the laser parameters are the same as in figure 1 .

charge $Z$ enters the calculation dynamically through the Sommerfeld parameter $\xi$ (in contrast, the FBA cross section is simply proportional to $Z^{2}$ ).

\section{Final remarks and conclusions}

Although many efforts were made to advance beyond the first-order Born approximation, most of them have a limited range (beyond FBA) of applicability at the expense of significant complications in the theory [9]. In this paper we investigated laser-assisted Mott scattering in the Coulomb approximation which is supposed to yield reasonable results for a laser-free case when the kinetic energy of the impact electron is far greater than its potential energy in the field of the target nucleus. At small angles, the results of the present treatment agree well with those of the FBA. With increasing scattering angles, however, we observe significant effects upon including the Coulomb field of the residual ion in the theory. In general the cross sections 


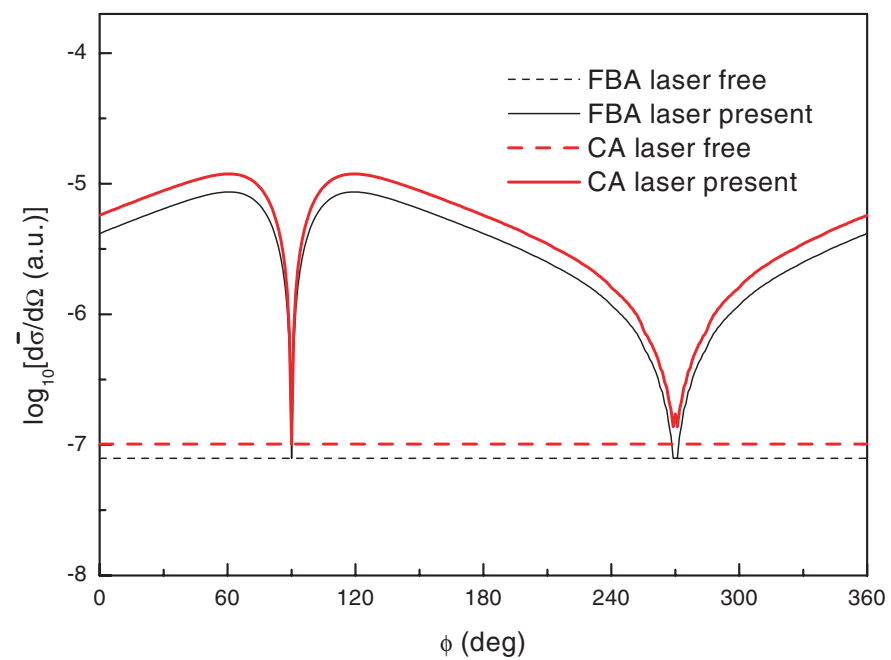

Figure 3. Azimuthal angular dependence of the summed differential cross section for the laser polarization geometry $\mathcal{E}_{0} \perp \mathbf{p}$ at the scattering angles $\theta=90^{\circ}$. The impact energy and the laser parameters are the same as in figure 1 .

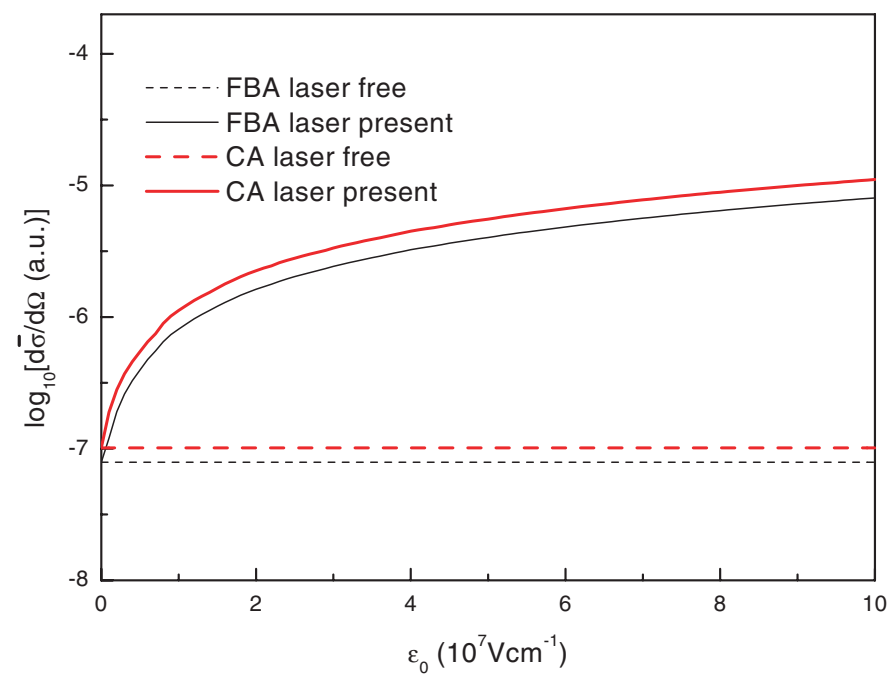

Figure 4. The field strength dependence of the summed differential cross section at $\theta=90^{\circ}, \phi=$ $0^{\circ}$ for the frequency $\hbar \omega=1.17 \mathrm{eV}$. The impact energy and the laser polarization direction are the same as in figure 1 .

evaluated within the Coulomb approximation are larger than the FBA predictions. In particular, the incorporation of the Coulomb ionic field leads to a qualitatively different dependence of the summed cross sections on the laser frequency in the high frequency regime: the CA results merge together with the laser-free results at much higher frequencies than the FBA results do. The discrepancies between CA and FBA are more noticeable in the photoemission processes (induced Bremsstrahlung) than in photoabsorption (inverse Bremsstrahlung). The dependencies of the differential cross section on other field parameters, on the impact energy and on the charge state of the residual ion have also been investigated. 


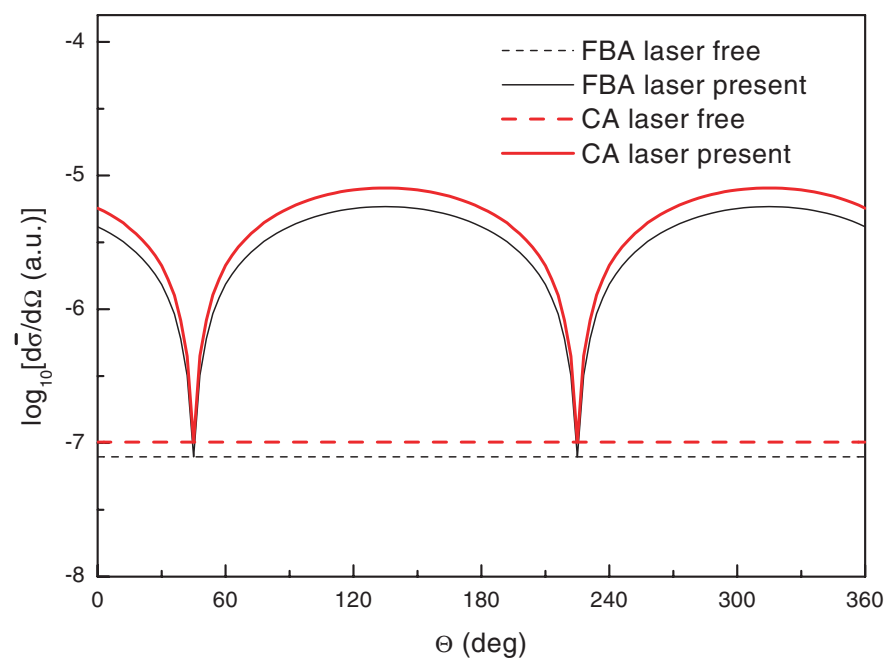

Figure 5. Summed differential cross sections versus the polar angle of the electric vector of the field for $\theta=90^{\circ}, \phi=0^{\circ}$. The impact energy and the laser parameters are the same as in figure 1 .

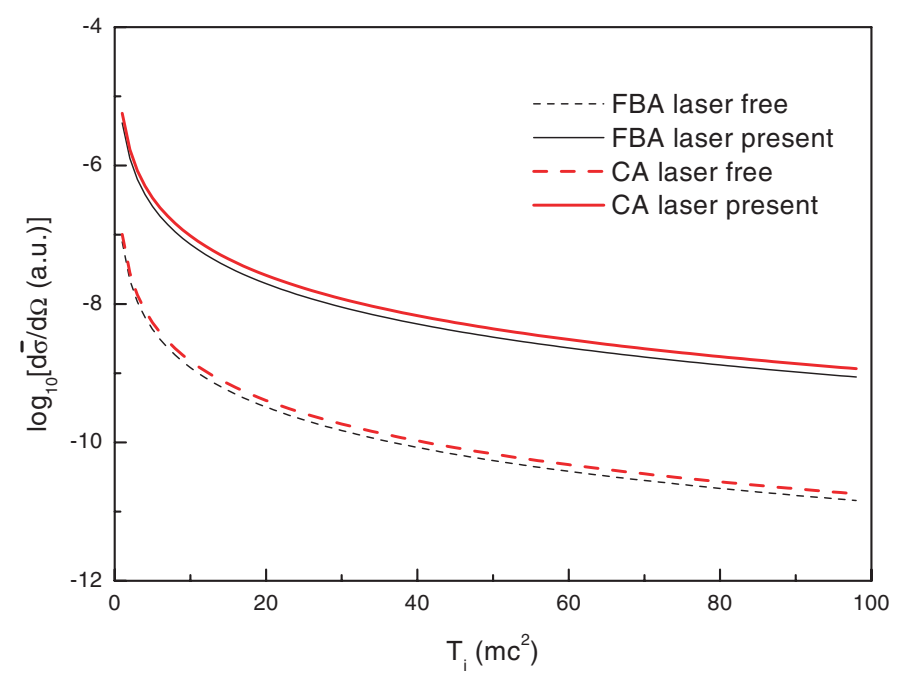

Figure 6. Summed differential cross sections for $\theta=90^{\circ}, \phi=0^{\circ}$ versus the kinetic energy of the incident electron. The laser parameters and the laser polarization direction are the same as in figure 1 .

While the Coulomb-Volkov state is well known in non-relativistic strong laser physics [17-20], the accuracy of this approximation for the relativistic scattering remains an open question. In the absence of full $a b$ initio calculations and/or appropriate experiments that may judge the accuracy of this approximation one has to consider the present findings as a preliminary description of the laser-assisted, relativistic Mott scattering. One may, however, hope that for the medium laser intensities, as considered here, the CA approximation will be of comparable accuracy as in the laser-free case.

In the present study we utilized the prior form of the $S$-matrix. Formally, one may equally express the $S$-matrix in the post form where the final state is treated as a relativistic 


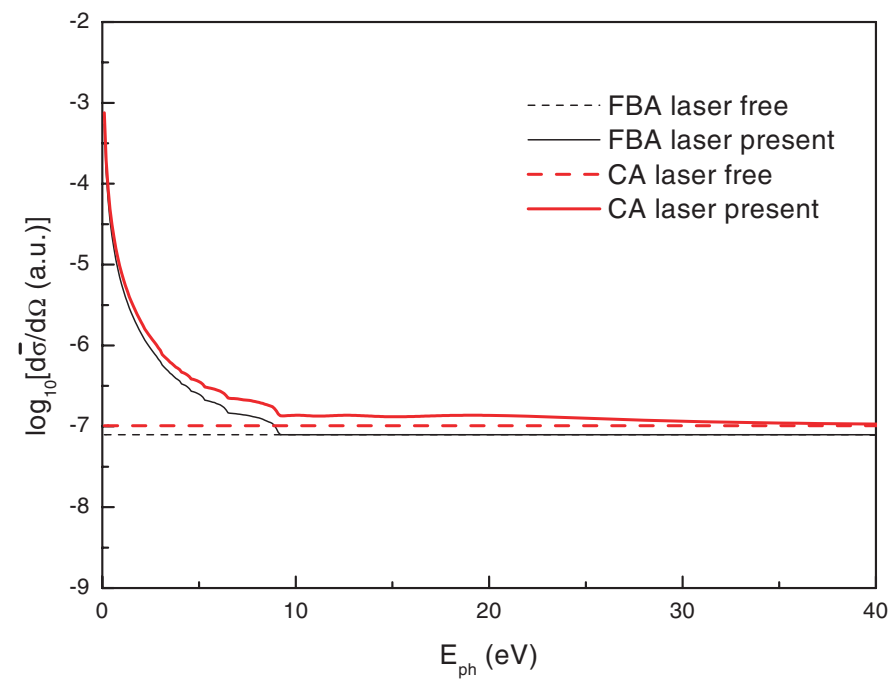

Figure 7. The photon energy (field frequency) dependence of the summed differential cross section for the scattering angles $\theta=90^{\circ}, \phi=0^{\circ}$. The field strength is $\mathcal{E}_{0}=5.14 \times 10^{7} \mathrm{~V} \mathrm{~cm}^{-1}$. The impact energy and the laser polarization direction are the same as in figure 1 .

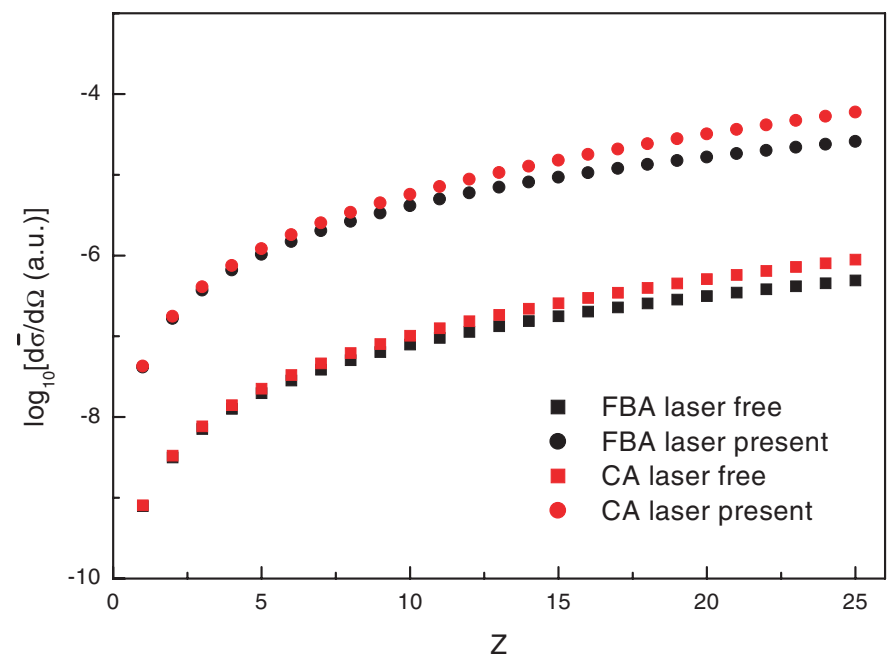

Figure 8. Summed differential cross sections versus nucleus charge $Z$. The scattering angles are $\theta=90^{\circ}, \phi=0^{\circ}$. The laser parameters, the laser polarization direction and the impact energy of electron are the same as in figure 1.

Coulomb-Volkov state. Generally speaking, for the laser-free case, both the prior and the post forms give exactly the same results for the cross sections when the spacetime integration in the $S$-matrix is performed. This is because the momenta and the energies of the initial and the final states are of the same magnitude. For the laser-assisted case, however, differences may occur between both forms due to the energy and momentum transfer between the colliding system and the laser field. But for the medium field intensity that we consider in the present calculation, no significant difference will occur between both forms. 


\section{Acknowledgments}

This work is supported by the National Natural Science Foundation of China under Grant numbers 10074060 and 10075043 . LSM would like to acknowledge the financial support from the DAAD during his stay in Germany.

\section{References}

[1] Milchberg H and Freeman R 1996 J Opt. Soc. Am. B 1351

[2] Joshi C et al 2002 Phys. Plasm. 91845

[3] Denisov M M and Fedorov M V 1967 Zh. Éksp. Teor. Fiz. 531340 Denisov M M and Fedorov M V 1968 Sov. Phys.-JETP 26779 (Engl. Transl.)

[4] Szymanowski C, Véniard V, Taïeb R, Maquet A and Keitel C H 1997 Phys. Rev. A 563846

[5] Szymanowski C and Maquet A 1998 Opt. Express 2262

[6] Panek P, Kamński J Z and Ehlotzky F 2002 Phys. Rev. A 65033408

[7] Li S-M, Berakdar J, Chen J and Zhou Z-F 2003 Phys. Rev. A 67063409

[8] Rosenberg L and Zhou F 1991 J. Phys. A: Math. Gen. 24631 Zhou F and Rosenberg L 1992 Phys. Rev. A 457818

[9] Roshchupkin S P 1993 Laser Phys. 3414

[10] Hovhannisyan T R, Markossian A G and Mkrtchian G F 2002 Eur. Phys. J. D 2017

[11] Berestetskii V B, Lifshitz E M and Pitaevskii L P 1982 Quantum Electrodynamics (Course of Theoretical Physics Vol 4) 2nd edn (Oxford: Reed Educational and Professional Publishing)

[12] Abramowitz M and Stegun I A (ed) 1984 Pocketbook of Mathematical Functions (Frankfurt a.M.: Verlag Harri Deutsch)

[13] Gradshteyn I S and Ryzhik I M 1980 Tables of Integrals, Series, and Products (New York: Academic)

[14] Akhiezer A I and Merenkov N P 1996 J. Phys. B: At. Mol. Opt. Phys. 292135

[15] Akhiezer A I and Berestetsky V B 1969 Quantum Electrodynamics (Moscow: Nauka)

[16] Greiner W and Reinhardt J 1994 Quantum Electrodynamics 2nd edn (Berlin: Springer)

[17] Jain M and Tzoar N 1978 Phys. Rev. A 18538

[18] Cavaliere P, Ferrante G and Leone C 1980 J. Phys. B: At. Mol. Phys. 134495

[19] Li S-M, Chen J and Zhou Z-F 2002 J. Phys. B: At. Mol. Opt. Phys. 35557

[20] Kornev A S and Zon B A 2002 J. Phys. B: At. Mol. Opt. Phys. 352451 